\title{
Contribución económica de la pesca artesanal a la economía de las comunidades ribereñas del Amazonas colombiano
}

\section{Economic contribution of the traditional fishing to the economy of the riverside communities of the Colombian Amazon}

\section{Contribuição econômica da pesca artesanal à economia das comunidades ribeirinhas da Amazônia colombiana}

\author{
Catalina Trujillo Osorio \& Adriana María Flórez Laiseca² \\ 'Economista, Magister en Estudios Amazónicos. Economista, Especialista Tecnológica \\ en Evaluación de Impacto Ambiental, Magister en Medio Ambiente y Desarrollo Sostenible \\ 1, 2Programa de Economía, Facultad de Ciencias Económicas, Universidad \\ del Quindío. Armenia, Quindio, Colombia \\ 1'ctrujillo@uniquindio.edu.co, 2amflorez@uniquindio.edu.co
}

\section{Resumen}

Este artículo de investigación analiza la importancia de la pesca artesanal para la economía y el bienestar de los hogares que habitan la ribera del rio Amazonas. Usando diarios de consumo y producción, se registró información sobre ingresos diarios de 23 hogares, en dos comunidades ribereñas de la Amazonia colombiana. Mediante la aplicación de métodos cuantitativos se encontró que el $78 \%$ de los ingresos familiares dependen del aprovechamiento de los recursos naturales, el $24 \%$ se deriva de la pesca artesanal, exclusivamente, y el $81 \%$ del valor de la ingesta de proteína la constituye el consumo de pescado. Los resultados obtenidos dan cuenta de la importancia social y económica de los ecosistemas pesqueros, y visibilizan la necesidad de implementar acciones de conservación y gestión ambiental y pesquera para garantizar la oferta natural del recurso, y por esta vía el sustento, la seguridad alimentaria y el bienestar de las comunidades ribereñas.
Palabras clave: autoconsumo, comunidad ribereña, economía indígena, ingresos ambientales, recurso pesquero.

\section{Abstract}

This research article analyzes the importance of the traditional fishing for the economy and the welfare of them homes that inhabit the bank of the river Amazon. Using diaries of consumption and production, there was information about daily income of 23 homes, two riverside communities of the Colombian Amazon. Through the application of quantitative methods found that $78 \%$ of family income are dependent on the exploitation of natural resources, $24 \%$ is derived from fishing, exclusively, and $81 \%$ of the value of the intake of protein is fish consumption. The results give account of the social and economic importance of fisheries ecosystems, and make visible the need to implement actions of conservation and environmental management and fishing to 
ensure the natural supply of the resource, and this via the livelihood, food security and the well-being of coastal communities.

Key-words: personal consumption, riverside community, indigenous economy, environmental income, fishing resource.

\section{Resumo}

Este artigo de pesquisa analisa a importância da pesca artesanal para a economia e supervivência dos lares que habitam a beira do rio Amazonas. Usando diárias de consumos e produção foi registrada informação sobre ingressos diários de 23 lares em duas comunidades ribeirinhas da Amazônia colombiana. Aplicando-se métodos quantitativos foi encontrado que $78 \%$ dos ingressos familiares dependem do aproveitamento dos recursos naturais, o $24 \%$ vem da pesca artesanal exclusivamente, e $81 \%$ do valor da ingesta da proteína vem do consumo de peixe. Os resultados obtidos mostram a importância social e econômica dos ecossistemas pesqueiros e deixam ver a necessidade de implementar ações de conservação e gestão ambiental e pesqueira para garantir a oferta natural do recurso, e assim o sustento, a segurança alimentar e o bem-estar das comunidades da beira do rio Amazonas.

Palavras-chave: autoconsumo, comunidade ribeirinha, economia indígena, ingressos ambientais, recurso pesqueiro.

\section{Introducción}

Históricamente la pesca de agua dulce y el recurso pesquero han constituido un elemento de gran importancia en las sociedades rurales y en la economía local, regional y mundial (Morán, Téllez, \& Cifuentes, 2010; Pinedo \& Soria, 2008); no obstante su importancia en Colombia nunca se ha visibilizado la magnitud del recurso, ni tampoco se ha reconocido como un sector económico relevante y significativo dentro de las economías locales, regionales o nacionales (Agudelo, Alonso \& Sánchez, 2009; Domínguez, 2005; Fernández, 1996; Zamora, Narváez, \& Londoño, 2007); Las razones para este modesto tratamiento se encuentran en la naturaleza misma de la actividad y las sociedades que la practican, caracterizadas generalmente por su alta ruralidad, tradición e informalidad. Estas características socioculturales de la pesca de agua dulce, la convierten en un recurso invisible para la economía monetaria y al mismo tiempo, la hace un elemento invaluable e irremplazable para las economías locales tradicionales, que a su vez viven y dependen de él para su subsistencia (Camacho, 2012; Duque et al., 2008; Durrance, 2003a; Trujillo, 2008; Meisel, Bonilla, \& Sánchez, 2013; Vieco \& Oyuela, 1999; Zamora, Narváez, \& Londoño, 2007).
En este contexto cabe entonces preguntarse ¿en términos económicos, qué tan representativa es la pesca artesanal para las comunidades rurales-ribereñas donde predomina la economía de subsistencia?; ¿cuál es el nivel de dependencia de estas sociedades del recurso pesquero y en efecto de su estado de conservación?; ¿cuál es el grado de contribución del río y otros cuerpos de agua en la economía de estos hogares y a su desarrollo social y cultural?; finalmente, en qué medida se ven afectadas estas sociedades ante los actuales fenómenos de deterioro ambiental y cambio climático?

En consecuencia, vale la pena contextualizar a través del caso de la Amazonia colombiana, sobre las aguas del río Amazonas, donde se observa una sociedad ribereña que depende del recurso pesquero como su principal fuente de vida, tanto para el consumo como para la venta y la dinamización de la economía de toda la región sur del trapecio amazónico. En efecto, esta realidad no aparece visible ni para la sociedad, ni para las instituciones regionales, y por ende, el recurso no es valorado, ni protegido (Fabré \& Alonso, 1998; 
Duque et al., 2008; Camacho, 2012; Ochoa, Wood \& Zárate, 2006; Trujillo, 2014). En la actualidad, la región experimenta un sistema económico dual donde coexisten dos sistemas económicos antagónicos; el sistema de la economía tradicional (o de subsistencia) caracterizado por actividades de horticultura, cacería, pesca y extracción para el consumo directo a partir del uso de los recursos naturales, y el sistema económico moderno o de mercado, caracterizado por la producción de excedentes a partir de la extracción de recursos naturales para la generación de ingresos monetarios (Murillo, 2006; Rodríguez \& Maldonado, 2012; Trujillo, 2008).

Esta dualidad económica ofrece ciertas ventajas a la población ribereña, pues la integración al mercado les facilita el acceso a bienes y dinámicas modernas y también les permite decidir sobre su educación y modo de vida; pero al mismo tiempo esta doble economía ha repercutido en la forma en que se utilizan los recursos y en consecuencia en la calidad ambiental, la seguridad alimentaria, la economía doméstica y la calidad de vida de los pobladores ribereños. (Agudelo, Sánchez, Rodríguez, Bonilla \& Gómez, 2011; Huérfano, 2010; Pantevis, 2013; Trujillo, 2008).

Este artículo de investigación tiene como objetivo estimar la importancia de la pesca artesanal dentro de la economía de una comunidad ribereña asentada en el margen del río Amazonas, sobre la desembocadura del rio Loretoyacu, en el municipio de Puerto Nariño, en la Amazonia colombiana.

\section{Marco metodológico}

La información que se presenta, hace parte de una investigación sobre producción, venta y consumo de recursos naturales, entre ellos el recurso pesquero, realizada en comunidades ribereñas del sur del Trapecio Amazónico. En este artículo sólo se analizarán las comunidades que hacen uso directo de los ríos Amazonas, Loretoyacu, y el sistema lacustre de Tarapoto, en el municipio de Puerto Nariño, departamento del Amazonas, Colombia, y se identifica la contribución de la pesca artesanal a la economía de los hogares de estas comunidades. Las labores de campo se realizaron a partir de la convivencia y trabajo directo con la población objeto de estudio, que a su vez permitió llevar a cabo un monitoreo diario para identificar los diferentes recursos que aportan la selva y los ríos a la economía doméstica. La cuantificación de estos productos, se realizó con base en el valor monetario de todos los bienes y servicios obtenidos por el grupo doméstico durante un período de tiempo fijo (todo tipo de productos derivados de la selva, ríos, lagos y del mercado). Este valor constituye una medida del ingreso total del hogar, que representa el valor de la producción del grupo doméstico, ya sea en dinero o en especie, con destino al consumo familiar, la redistribución social, el intercambio o la venta (Cavendish, 1999).

\section{Contexto socio-ambiental del municipio de Puerto Nariño}

Puerto Nariño es un pequeño municipio del sur de la Amazonia colombiana (Figura 1), ubicado entre los ríos Cotué, Putumayo Amazonas y Atacuári (entre $03^{\circ} 54^{\prime}$ y $03^{\circ} 12^{\prime} \mathrm{S}$, y $70^{\circ} 17^{\prime}$ y $70^{\circ}$ $42^{\prime}$ W). Cuenta con una cabecera municipal, que lleva su mismo nombre y una zona rural ocupada principalmente por el Resguardo Indígena Ticoya, algunas parcialidades indígenas y el Parque $\mathrm{Na}$ cional Natural Amacayacu. La vía de acceso a los asentamientos humanos es exclusivamente fluvial; el principal eje navegable es el rio Amazonas, que comunica al municipio con poblaciones peruanas vecinas y con el municipio de Leticia a través del cual desarrollan sus actividades comerciales (Gobernación del Amazonas, 2012). 


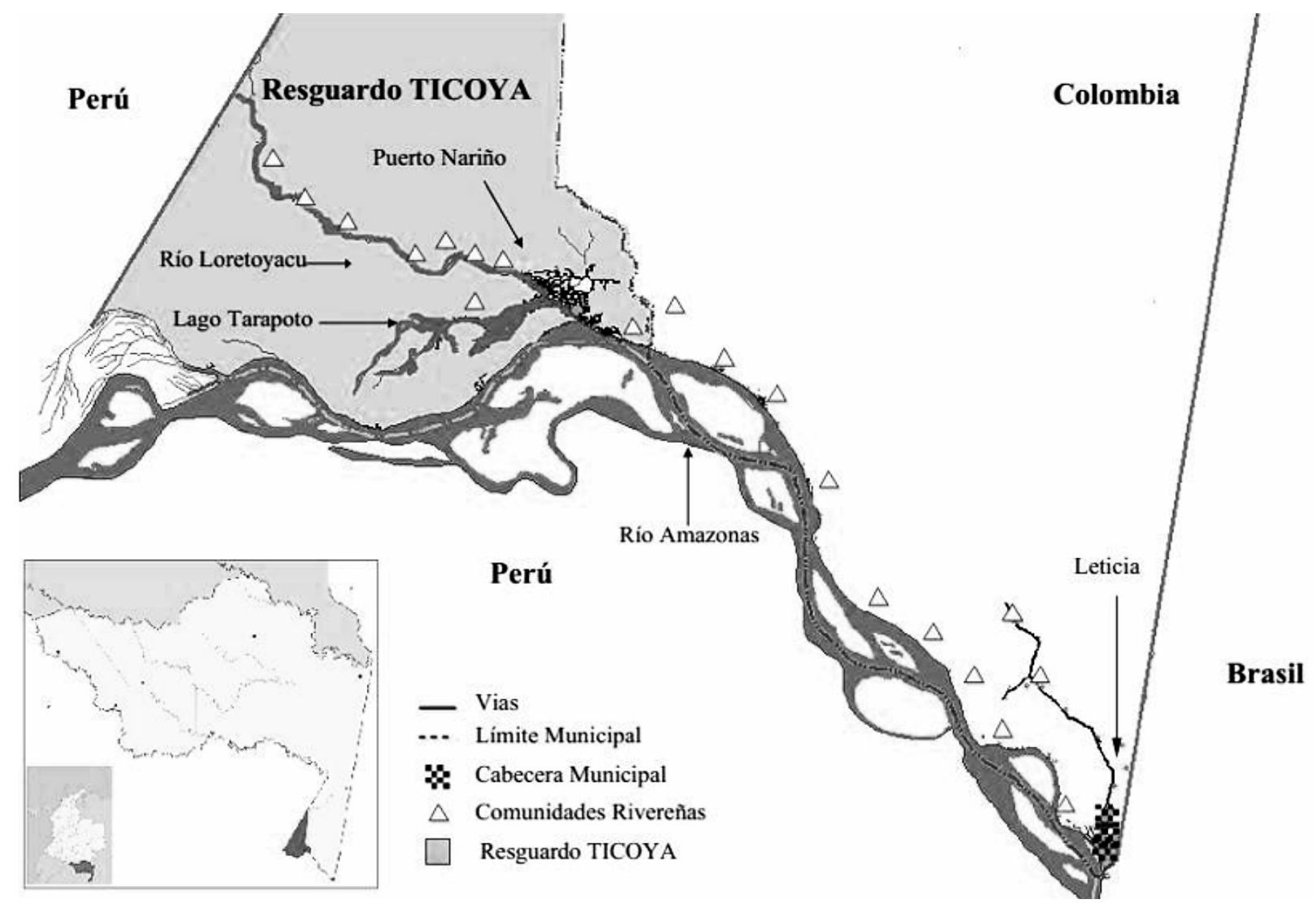

Figura 1. Trapecio amazónico, ubicación de Puerto Nariño

Fuente: Adaptado de Gobernación del Amazonas, 2012.

El municipio cuenta con un área aproximada de 154.160 ha, donde el 0,04\% representan la cabecera municipal, el $0,98 \%$ es área rural municipal y el restante $92 \%$ del área pertenece al resguardo Ticoya. Para el año 2011, el municipio albergaba una población de 5.699 habitantes con 1.769 (31\%) asentados en la cabecera (Alcaldía de Puerto Nariño, 2012); y para el año 2015 la población se encontraba en 6.163 habitantes, con 1.907 (30\%) radicados en la cabecera. En los últimos veinte años, tanto el municipio como el casco urbano, han registrado un importante crecimiento poblacional. Como se reporta en la Tabla 1, el municipio ha experimentado un crecimiento poblacional de $53 \%$ durante los últimos 20 años. Este crecimiento demográfico se ha dado por los cambios en los modos de vida de la población y la tendencia a migrar a las cabeceras en busca de servicios y mercados; adicionalmente a nivel local se presenta una alta tasa de natalidad con 28 nacimientos por cada 1000 habitantes, muy superior a la media nacional, de 19 nacimientos por cada 1000 habitantes para el mismo periodo (DANE, 2011). Estas dinámicas sociales y culturales junto a los nuevos modos de vida y consumo, que exigen las cabeceras, constituyen un reto y una amenaza para la sostenibilidad cultural, económica y ambiental del municipio, como lo señalan Meisel, Bonilla, \& Sánchez (2013) en su estudio sobre la geografía económica de la Amazonia colombiana. 
Tabla 1. Población Urbana y Rural de Puerto Nariño (1995-2015)

\begin{tabular}{l|c|c|c|c|c|}
\hline Población municipal por área & 1995 & 2000 & 2005 & 2011 & 2015 \\
\hline Población Cabecera & 1.346 & 1.711 & 1.805 & $1.769^{\mathrm{a}}$ & $1.907^{\mathrm{b}}$ \\
\hline Población Rural & 2.661 & 3.008 & 3.583 & $3.930^{\mathrm{a}}$ & $4.258^{\mathrm{b}}$ \\
\hline Población total & 4.007 & 4.719 & 5.388 & $5.699^{\mathrm{a}}$ & $6.163^{\mathrm{b}}$ \\
\hline Proyección DANE & n.a & n.a & $6.938^{\mathrm{c}}$ & $7.691^{\mathrm{c}}$ & $8.162^{\mathrm{c}}$ \\
\hline
\end{tabular}

Fuente: Adaptado de Ochoa, Wood, \& Zárate, 2006.

${ }^{a}$ Gobernación del Amazonas (2012)

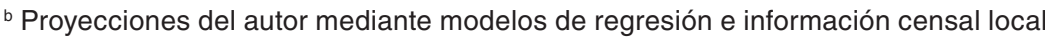

${ }^{9}$ DANE (2011). Proyecciones de población municipal por área

Las actividades económicas del municipio son principalmente de tipo extractivista, predominando la pesca, la extracción maderera, el uso de productos del bosque y en menor medida el turismo (Murillo, 2006; Rodríguez \& Maldonado, 2009). Las fuentes de empleo son limitadas dependiendo de las temporadas de turismo y otras actividades intermitentes asociadas a los ciclos políticos y de contratación del sector público. El mercado local está en proceso de consolidación; no obstante existe ausencia de mercados, causando una ineficiencia en el sistemas de comercio actual, toda vez que no satisface las demandas de consumo de la población local, ni la generación de nuevas alternativas de empleo (Alcaldía de Puerto Nariño, 2012). La población se conforma por indígenas Ticunas, Cocamas, Yaguas, colonos y una nueva generación de habitantes resultado de la mezcla poblacional. Culturalmente, la población se caracteriza por su tradición indígena pesquera y su relación con el recurso hídrico, toda vez que tradicionalmente ha habitado las orillas de los ríos y cuenta con gran conocimiento y manejo de las dinámicas de ciclo hídrico y pesquero. (Durrance, 2003a; Vieco \& Oyuela, 1999).
El municipio está conformado en su mayoría por el territorio del Resguardo Ticoya que ocupa el 92\% del área del municipio (Alcaldía de Puerto Nariño, 2008). En la actualidad los resguardos indígenas son una forma de propiedad colectiva que representa una figura de conservación ambiental y cultural donde se mantienen las economías de subsistencia tradicionales y la producción para el autoabastecimiento, la seguridad alimentaria y la reproducción social (Trujillo \& Escobar, 2015); sin embargo, la presión demográfica y la presión pesquera están iniciando un proceso de deterioro ambiental de los recursos hídricos dentro del resguardo (Agudelo, Alonso, \& Sánchez, 2009; Trujillo \& Duque, 2014). El territorio del resguardo está conformado por $142.483 \mathrm{ha}$, incluyendo el área bañada por los lagos y humedales de Tarapoto, que constituyen el principal recurso hidrobiológico de la zona y los principales ambientes pesqueros de la población ribereña. Los lagos (Figura 2) se localizan al interior del resguardo y están conformados por once (11) sistemas lacustres (Tarapoto Redondo y Tarapoto largo, El Sapo, El Correo, Chirillo, Chimbillo, Igarapeguazú, Umaricocha, Chepeté, Cochalarga) que ocupan una extensión de 82.000 ha y representan el $8,9 \%$ del territorio total del resguardo durante la época de mayor caudal del ciclo hídrico (Trujillo \& Duque, 2014). 


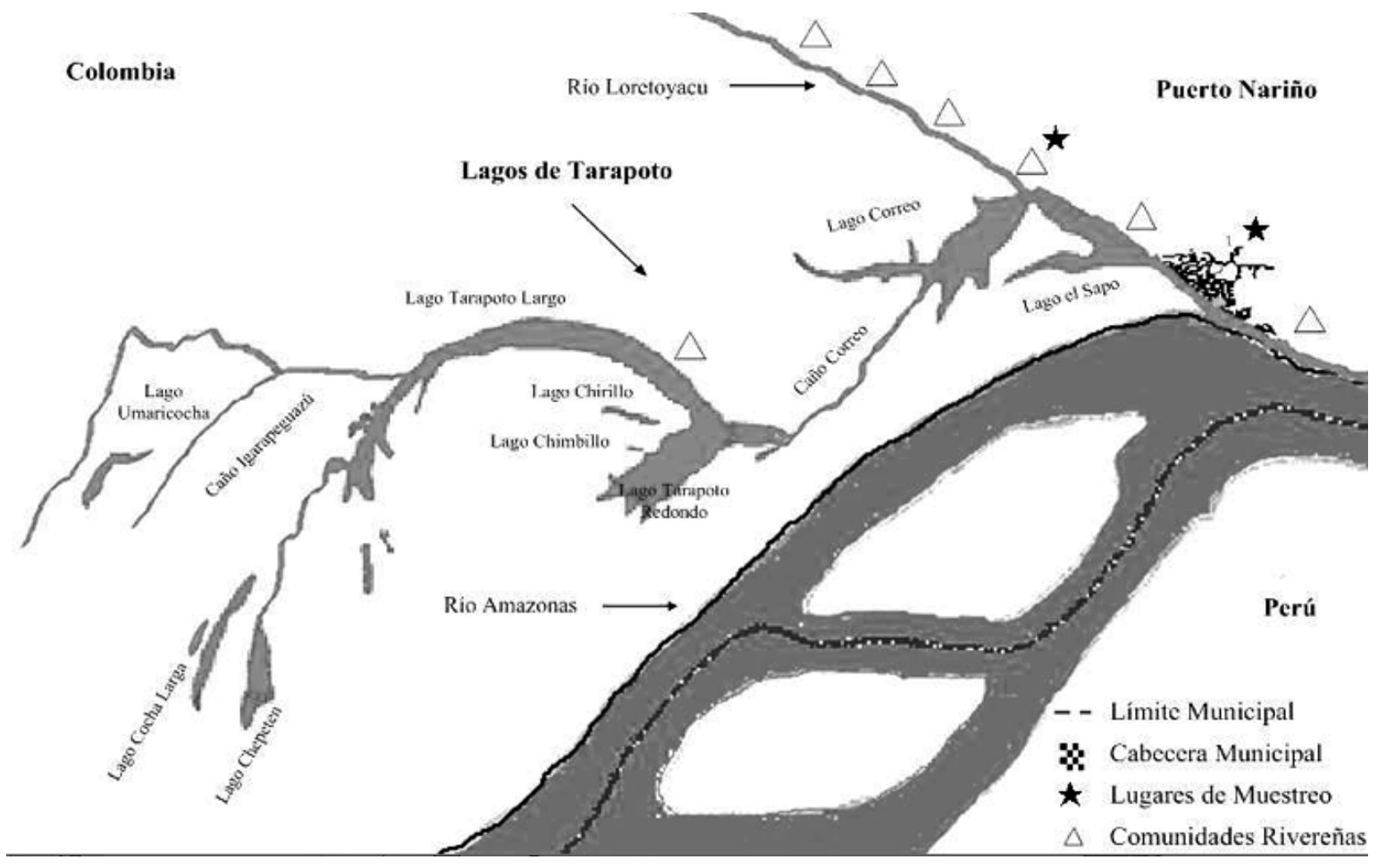

Figura 2. Ambientes pesqueros del municipio de Puerto Nariño

Fuente: Adaptado de Trujillo \& Duque, 2014.

Por su parte, el ciclo hídrico (o época de creciente y sequía, o aguas altas y bajas) constituye la dinámica periódica entre la creciente y el descenso del nivel hidrológico de los ríos, lagos, y demás cuerpos de agua (Trujillo \& Trujillo, 2010). Este fenómeno está determinado por las lluvias ocurridas sobre la cordillera de los Andes y determina toda la dinámica del recurso pesquero, tanto en su ciclo de vida como en sus patrones alimenticios, y por consiguiente el nivel de oferta natural del recurso, pero también los periodos en que éste está expuesto a una mayor vulnerabilidad (Ardila, 2009; Durrance ${ }^{\text {b }}$ 2003; López, 2008).

Actualmente, y a raíz de su cercanía con la cabecera municipal los ambientes pesqueros del resguardo Ticoya y el municipio, sufren una fuerte presión pesquera, generada tanto por la pesquería de subsistencia como por otras actividades como el turismo, la extracción de especies maderables, la destrucción del bosque inundado y los corredores de migración y alimentación de las diversas especies acuáticas, poniendo en riesgo la suficiencia económica y alimentaria de esta población ribereña. (Rodríguez \& Maldonado, 2012; Trujillo \& Duque, 2014).

\section{Aspectos metodológicos para la valoración de} los ingresos ambientales en hogares ribereños

Para el desarrollo de este trabajo se utilizó la información recopilada dentro de un proyecto marco orientado a la cuantificación de los ingresos por bienes y servicios ambientales en resguardos indígenas del Trapecio Amazónico. La información fue recopilada mediante monitoreo diario a las actividades de producción, pesca, consumo y comercialización de los hogares participantes en dos comunidades de Puerto Nariño (la comunidad indígena de San Francisco de Loretoyacu, y el casco urbano). Para el monitoreo, se tomó el hogar como unidad de estudio, y cada una de ellas recopiló y aportó su propia información económica. Estos métodos fueron diseñados y ajustados en una comunidad del municipio, donde durante dos meses se desarrollaron pruebas y ajustes, hasta asegurar la efectividad y la viabilidad de aplicación de cada uno de los instrumentos de muestreo. 
Medición del ingreso del hogar: Se entiende por Ingreso del hogar todas las entradas de recursos que este recibe, durante un periodo de tiempo determinado, ya sea para el consumo, la venta o la redistribución de productos entre el grupo social
(Cavendish, 1999). Para cuantificar los diferentes componentes y categorías del ingreso se utilizó la unidad monetaria (dinero) como unidad de medida. La Figura 3. presenta la composición del ingreso bajo un enfoque sistémico.

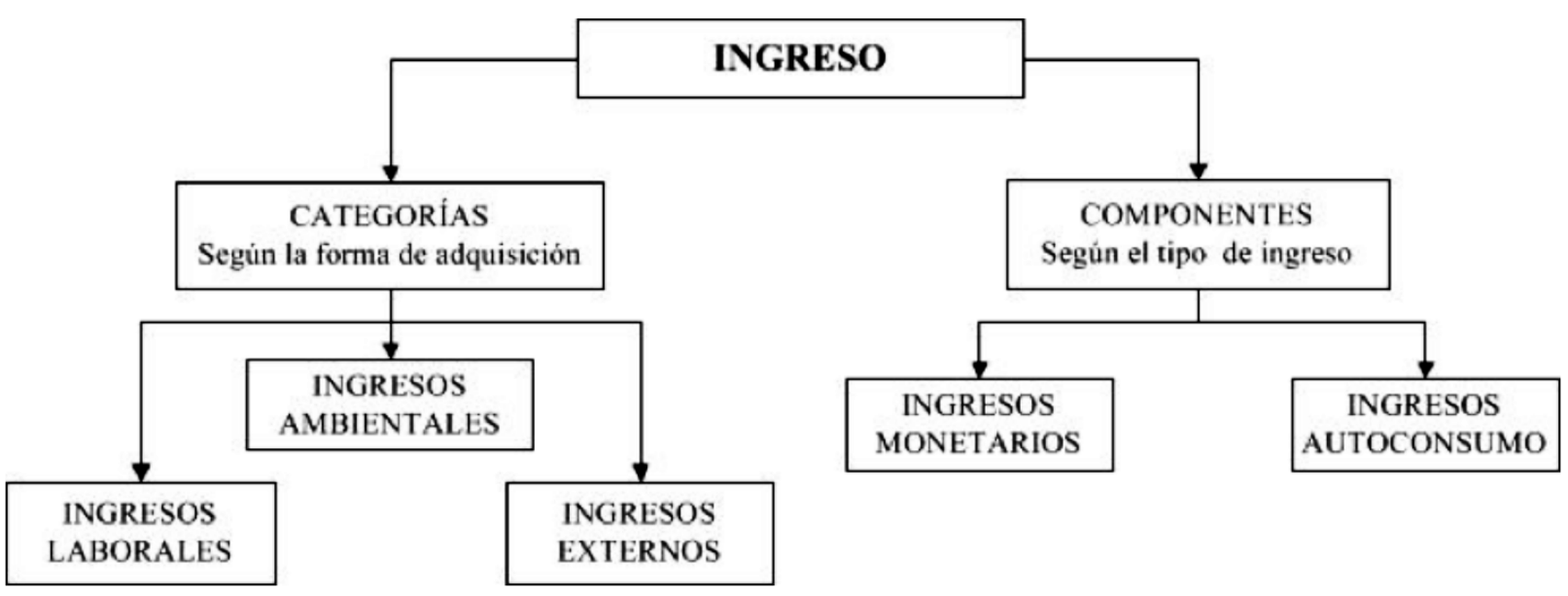

Figura 3. Composición del ingreso

Fuente: Adaptado del modelo conceptual de Cavendish, 1999.

La Figura 3. representa la composición teórica del ingreso total real y las dimensiones que lo conforman. Según la metodología desarrollada por William Cavendish de Oxford University, los ingresos que percibe un hogar se clasifican de dos formas: i) en subcomponentes según el tipo de ingreso y ii) en categorías según su forma de adquisición:

i) Los subcomponentes del ingreso dividen el ingreso total en dos tipos de ingreso; los Ingresos por Autoconsumo o en especie, que representan el valor de los alimentos y otros productos de la selva y el río producidos y consumidos directamente por los miembros del grupo doméstico; y los Ingresos Monetarios que reflejan el valor del ingreso en dinero obtenidos por la venta de productos, servicios, y la fuerza de trabajo. ii) Las categorías de ingreso representan las diferentes fuentes o sectores productivos generadores de ingreso. Para el caso de la economía en sociedades ribereñas se encontraron tres categorías: Ingresos Ambientales, derivados del uso de los recursos naturales; Ingresos laborales derivados de la venta de la fuerza de trabajo; e Ingresos externos provenientes de transferencias externas.

Estas dos clasificaciones conforman una matriz de ingresos (Figura 4) que refleja el valor de los ingresos monetarios, más el valor de los productos y servicios consumidos o usados directamente por el hogar durante un periodo contable, mediante las diversas formas de adquisición ya sea a través del aprovechamiento de los recursos naturales y el uso de los bienes y servicios ambientales, la venta de su mano de obra, o la obtención de ingresos por fuentes diferentes a las anteriores. 


\begin{tabular}{|c|c|c|c|c|}
\hline \multicolumn{5}{|c|}{ Componentes } \\
\hline \multirow{5}{*}{ 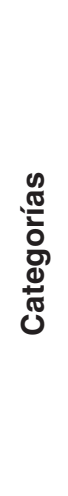 } & & $\begin{array}{l}\text { Ingresos por Autoconsumo } \\
\text { (En Especie) }\end{array}$ & $\begin{array}{l}\text { Ingresos por Venta } \\
\text { (Monetarios) }\end{array}$ & $\begin{array}{l}\text { Ingreso Total por Cate- } \\
\text { goria }\end{array}$ \\
\hline & Ambientales & $\begin{array}{l}\text { Valor del autoconsumo } \\
\text { familiar }\end{array}$ & $\begin{array}{l}\text { Venta de Bienes y Servicios } \\
\text { Ambientales }\end{array}$ & $\begin{array}{l}\text { Ingresos } \\
\text { Ambientales }\end{array}$ \\
\hline & Laborales & Salarios en especie & Salarios en dinero & Ingresos Laborales \\
\hline & Externos & $\begin{array}{l}\text { Valor de la redistribución } \\
\text { social recibida }\end{array}$ & $\begin{array}{l}\text { Subsidios, giros } \\
\text { remesas recibidas }\end{array}$ & Ingresos Externos \\
\hline & $\begin{array}{l}\text { Ingreso Total } \\
\text { por Componente }\end{array}$ & $\begin{array}{l}\text { Ingresos } \\
\text { por Autoconsumo }\end{array}$ & $\begin{array}{l}\text { Ingresos } \\
\text { Monetarios }\end{array}$ & $\begin{array}{l}\text { Ingreso Total Real } \\
\text { del Hogar }\end{array}$ \\
\hline
\end{tabular}

Figura 4. Matriz del Ingreso Total Real según Componentes y Categorías

Fuente: Adaptado del modelo conceptual de Cavendish (1999).

Medición de los ingresos ambientales: Al igual que los "Ingresos del Hogar", los ingresos ambientales son todos aquellos productos derivados del uso, manejo o extracción de un recurso natural, que "ingresan" al hogar, mediante alguna de las formas descritas; los ingresos ambientales entran al hogar en una de seis maneras a saber; mediante producción en chagras y rastrojos, recolección de productos del bosque, pesca, compra, intercambios o trueques, o en forma de regalos recibidos de terceros. De la misma forma los ingresos ambientales son utilizados para ser consumidos en el hogar, vendidos, intercambiados, regalados, o usados como insumos productivos. La Figura 5. presenta la matriz para la estimación de los ingresos ambientales, donde cada elemento de la matriz determina la clasificación del recurso según su uso final, ya sea como ingreso por venta, ingreso por consumo o como insumo productivo.

\begin{tabular}{|c|c|c|c|c|c|c|}
\hline \multicolumn{7}{|c|}{ Origen de los ingresos Ambientales } \\
\hline & & $\begin{array}{l}\text { Producción } \\
\text { y Recolección }\end{array}$ & Pesca & Compra & Intercambio & $\begin{array}{l}\text { Regalos } \\
\text { a la casa }\end{array}$ \\
\hline \multirow{4}{*}{ 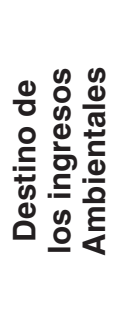 } & Consumo & Consumo & Consumo & Consumo & Consumo & Consumo \\
\hline & Venta & $\begin{array}{l}\text { Ingreso } \\
\text { monetario }\end{array}$ & $\begin{array}{l}\text { Ingreso } \\
\text { monetario }\end{array}$ & $\begin{array}{l}\text { Ingreso } \\
\text { monetario }\end{array}$ & $\begin{array}{l}\text { Ingreso } \\
\text { monetario }\end{array}$ & $\begin{array}{l}\text { Ingreso } \\
\text { monetario }\end{array}$ \\
\hline & Intercambio & Consumo & Consumo & Consumo & Consumo & Consumo \\
\hline & Regalo & Consumo futuro & Consumo futuro & Consumo futuro & Consumo futuro & $\begin{array}{l}\text { Consumo } \\
\text { futuro }\end{array}$ \\
\hline
\end{tabular}

Figura 5. Sistema contable de flujos de Ingresos Ambientales.

Para alimentar la matriz con información real, y a partir de ella valorar los ingresos ambientales de la sociedad de interés, se diseñó un instrumento específico para el registro y cuantificación de cada uno de los subcomponentes del ingreso. De esta manera, para cada cuadro de la matriz se utilizó un sistema de recolección de información específico. Se utilizaron tres diferentes instrumentos para la recolección de la información: i-diarios de producción y consumo, ii-cuestionario de caracterización económica, y iii- inventario de chagras y rastrojos (Trujillo, 2008). 
La medición de los ingresos ambientales derivados de la pesca artesanal: Se entiende que son todos los ingresos del hogar obtenidos a partir de la extracción, venta y consumo de un producto pesquero por parte del grupo familiar. Para el registro de los ingresos ambientales derivados de la pesca, se utilizó la sarta como unidad de medida estándar y se recopiló diariamente en cada hogar la cantidad de producto capturado por los integrantes del hogar, su lugar de captura y destino (consumo, venta, intercambio o regalo), y el precio de mercado del producto (la cantidad de dinero que debieran pagar por él), que para la fecha de estudio, y durante la época de aguas en ascenso se estimó en $\$ 3000$ pesos colombianos/sarta, en promedio, para las dos comunidades de estudio (Trujillo, 2008).

Instrumentos para la medición de los ingresos:

1. Cuantificación de los ingresos monetarios

Para estimar el valor de los ingresos en dinero cada hogar construyó un listado de sus actividades generadoras de ingreso, señalando el monto y la frecuencia de los mismos. Para el registro del ingreso se entrevistó a cada uno de los miembros del hogar mayor de 14 años de edad.

2. Cuantificación de los ingresos por consumo Para recopilar información detallada sobre la producción que cada hogar destina para el autoconsumo doméstico, se utilizó un sistema de registro donde cada hogar de la muestra, colectó de manera sistemática todos los alimentos consumidos diariamente por todos los miembros del grupo doméstico, durante el periodo de tiempo establecido. A través de este registro en "diarios de consumo" cada hogar participante suministró información detallada sobre el producto y la cantidad consumida, la forma de adquisición del producto, su precio en caso de haberlo comprado o el producto por el cual fue intercambiado dado el caso.

3. Cuantificación de los ingresos por redistribución Esta información se recopiló a través de los diarios de consumo dentro de la opción clasificada como "regalos al hogar" y a través del inventario de ingresos dentro de la sección "Transferencias".

\section{Análisis y presentación de la información}

Como el ingreso total del hogar representa el valor de la producción de todos los integrantes del grupo doméstico, los hogares con mayor población tenderán a presentar un mayor valor del ingreso, sin que esto signifique que el hogar perciba un mayor ingreso por integrante o que goce de una mayor disponibilidad de recursos. Por esta razón los indicadores de ingreso agregados deben ser ajustados por índices que reflejen las variaciones de las características demográficas al interior del hogar. De esta forma, para poder realizar comparaciones adecuadas del ingreso entre diferentes hogares, esta medida tuvo que ser corregida por:

1. Escalas de equivalencia (según la composición de los hogares por edad y género)

2. Economías de escala (según el tamaño de los hogares)

A partir de estos dos aspectos se construyó una nueva medida del tamaño del hogar conocida como Unidad de adulto Equivalente ajustada por economías de escala (UAE), estimada a partir de los coeficientes de las variaciones en el consumo de alimentos, generadas según cambia el tamaño del hogar. Esta metodología, desarrollada por (Deaton \& Grosh, 1989), es actualmente aplicada a los estudios económicos sobre análisis empírico del consumo de hogares. El índice de ajuste para corregir el ingreso del hogar según sus características internas se obtiene multiplicando el número de UAE por el coeficiente de cada hogar. Los resultados que se presentan a continuación se refieren a "el ingreso del hogar ajustado por UAE".

\section{Resultados y discusión}

\section{Análisis de la economía pesquera en comunida- des ribereñas.}

La pesca artesanal es la principal actividad socioeconómica de los pobladores de Puerto Nariño. A esta actividad se dedican durante el periodo de aguas bajas más del $50 \%$ de la población y una buena parte de las actividades económicas permanentes está directamente relacionada con el uso del 
recurso pesquero. Según estudios realizados para Puerto Nariño; (Ochoa, Wood, \& Zárate, 2006; Rodríguez \& Maldonado, 2009; Trujillo, 2008), en términos económicos y alimentarios, la pesca artesanal de subsistencia es la segunda actividad económica más relevante para la región incluyendo la pesca comercial, pues no sólo involucra una mayor población, sino que además garantiza la ingesta de proteína animal y la seguridad alimentaria de la población.

En términos económicos la producción y los ingresos de las sociedades de subsistencia tienen dos finalidades; garantizar la alimentación y necesidades directas de la familia, y generar excedentes de valor comercial que permitan complementar los bienes de consumo que no son producidos al interior del hogar (Gashe \& Vela, 2011). En la población ribereña de Puerto Nariño se encontró que la pesca responde por aproximadamente el $24 \%$ del valor total del ingreso familiar; de este el $46 \%$ de su valor se consumió directamente dentro del hogar, y el 54\% se destinó a la comercialización, como se describe en la Tabla 2.

Tabla 2. Composición de los ingresos del hogar ribereño de Puerto Nariño

\begin{tabular}{|c|c|c|c|}
\hline \multirow{3}{*}{$\begin{array}{l}\text { Actividades productivas } \\
\text { generadoras de ingresos } \\
\text { en hogares ribereños }\end{array}$} & \multicolumn{2}{|c|}{ Valor de la Producción } & \multirow{2}{*}{$\begin{array}{c}\text { Valor total de la producción } \\
\text { Ingreso total del hogar }\end{array}$} \\
\hline & Destinada al autoconsumo & Destinada a la Venta & \\
\hline & Fuente de Alimentación & Fuente de ingreso & (Alimentación + Ingreso) \\
\hline Cultivo en Chagras y solares & $18 \%$ & $14 \%$ & $31 \%$ \\
\hline Pesca & $14 \%$ & $10 \%$ & $24 \%$ \\
\hline Recolección de leña y madera & $8 \%$ & $7 \%$ & $15 \%$ \\
\hline Cacería & $3 \%$ & $1 \%$ & $4 \%$ \\
\hline $\begin{array}{l}\text { Recolección de frutas } \\
\text { y semillas para artesanías }\end{array}$ & $1 \%$ & $3 \%$ & $4 \%$ \\
\hline Cría de animales de patio & $3 \%$ & $1 \%$ & $4 \%$ \\
\hline Empleos & - & $18 \%$ & $18 \%$ \\
\hline Total & $46 \%$ & $54 \%$ & $100 \%$ \\
\hline
\end{tabular}

"No incluye el valor de los ingresos recibidos por transferencias externas, programas de seguridad social, alimentaria o subsidios.

La Tabla 2 y la Figura 6, presentan la composición del ingreso de los hogares de Puerto Nariño y la participación de cada actividad productiva en el ingreso total familiar. Se observa cómo la actividad que más ingresos aporta a la economía doméstica es la producción agrícola en chagras, rastrojos y solares, que en promedio genera el $31 \%$ de los ingresos domésti$\cos (18 \%$ por autoconsumo y $14 \%$ por venta). La pesca ocupa el segundo reglón dentro de la economía de subsistencia, aportando el $24 \%$ de los ingresos, seguido de los empleos directos (o venta de mano de obra) que generan el $18 \%$, y el corte y recolección de leña para fogón que contribuye con un 15\% dentro de la economía del hogar. Otras actividades tradicionales como la recolección de frutos y semillas y la cacería, de igual manera se encuentran presentes en los ingresos, aunque en menor participación. Actividades económicas no tradicionales introducidas durante los últimos años por proyectos de seguridad social (RESA, Familias Guardabosques, Acción Social), ya comienzan a reportarse como fuente de ingresos domésticos, como es el caso de los animales de patio, la cría de especies menores y las actividades asociadas a las iniciativas de ecoturismo. 


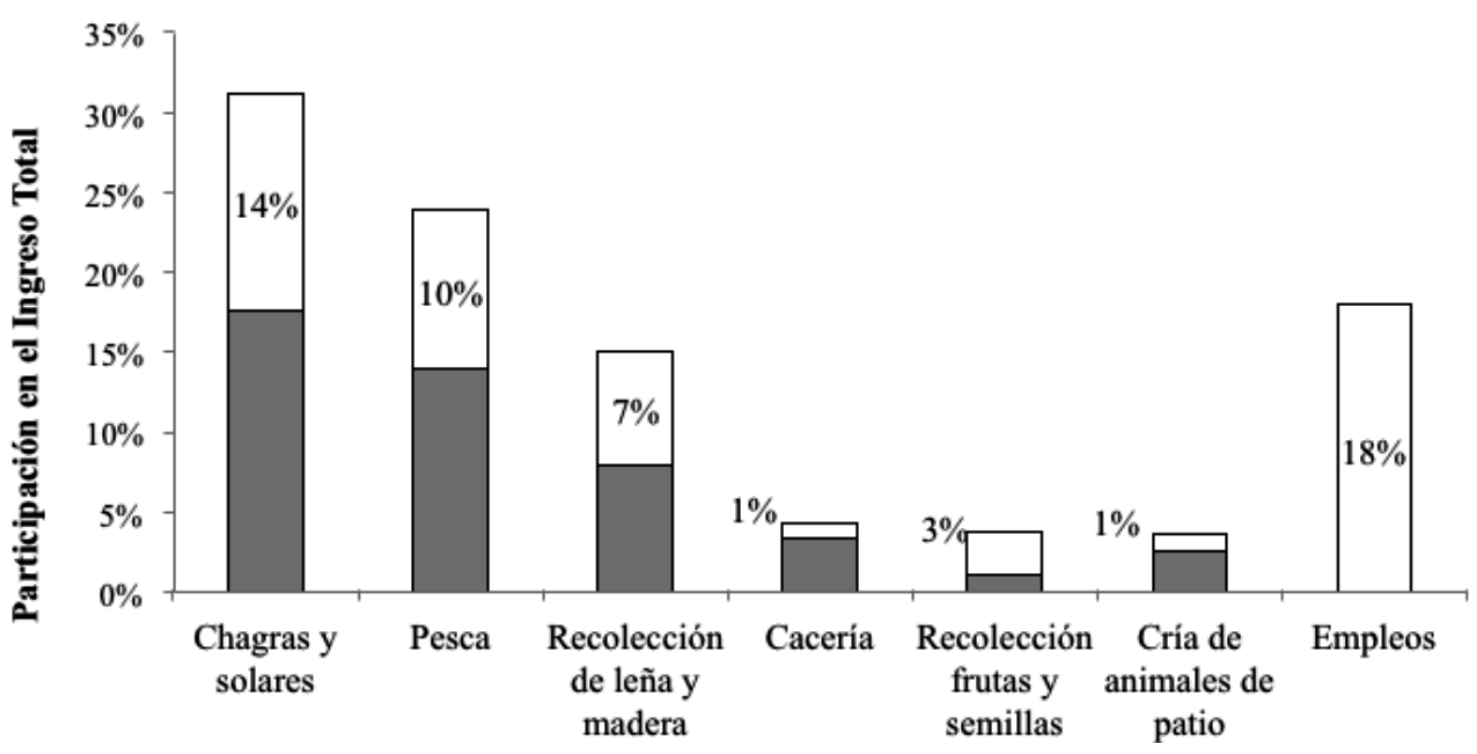

Figura 6. Composición de los ingresos del hogar ribereño de Puerto Nariño

Otras actividades tradicionales como la recolección de frutos y semillas y la cacería, de igual manera se encuentran presentes en los ingresos, aunque en menor participación. Actividades económicas notradicionales introducidas durante los últimos años por proyectos de seguridad social (RESA, Familias Guardabosques, Acción Social), ya comienzan a reportarse como fuente de ingresos domésticos, como es el caso de los animales de patio, la cría de especies menores y las actividades asociadas a las iniciativas de ecoturismo.

\section{La pesca para el consumo del hogar}

En referencia a lo anterior, se puede estimar que la pesca ocupa el segundo reglón más importante aportando el $24 \%$ de los ingresos dentro de la economía de subsistencia; de los cuales el 10\% corresponde a la venta y comercialización de productos obtenidos a partir del uso del recurso pesquero (es decir ingresos monetarios derivados de la pesca) y el $14 \%$ representa el valor del consumo de pescado dentro del grupo doméstico del pescador (es decir el valor del autoconsumo).

Adicionalmente, el autoconsumo de pescado aparece como la primera fuente de proteína animal en la dieta local y como la segunda fuente de alimentos después de la agricultura y horticultura (producción en chagras y rastrojos). En términos económicos, la pesca aporta el $14 \%$ del valor de los alimentos consumidos directamente dentro del grupo doméstico sin contar el valor de otros alimentos que son comprados con los ingresos monetarios obtenidos a partir de la venta del excedente pesquero. Así para el año 2007, para los hogares del área de Puerto Nariño (conformados por 4,7 UAE), el consumo mensual de pescado representaba un valor de $\$ 109.726$ (pesos colombianos) por hogar a precios de 2007, sin incluir el valor de otros productos alimentarios. Este consumo calculado a precios de 2015 representa un costo de $\$ 162.731$ pesos colombiano mensuales por hogar y $\$ 1.952 .781$ pesos colombianos al año. Este valor refleja el costo oculto del recurso pesquero que se consume dentro de una economía ribereña de subsistencia y representa la cantidad de dinero necesario para adquirir ese mismo producto en caso de no acceder a la pesca artesanal o de la pérdida de capacidad productiva del ecosistema. Esta cifra equivale al $84 \%$ de un salario mínimo (SMLV) tanto para año 2007 año en que se realizó el estudio, como para el año 2015 fecha en que se preparó este artículo. Estas cifras evidencian la importancia económica del recurso pesquero, los recursos acuáticos asociados y los ecosistemas de provisión que lo generan, dada la importancia del valor del recurso, dentro del consumo y la economía de los hogares ribereños. 
La Tabla 3 presenta el valor oculto de los recursos y productos que son producidos y consumidos directamente al interior del hogar ribereño. Este valor representa la contribución económica de los recursos naturales y los sistemas productivos tradicionales a la seguridad alimentaria de la población ribereña.

Tabla 3. Fuentes y valores del autoconsumo del hogar ribereño a precios 2007.

\begin{tabular}{|c|c|c|c|}
\hline \multirow{2}{*}{$\begin{array}{l}\text { Actividades productivas } \\
\text { generadoras de ingresos } \\
\text { en hogares indígenas }\end{array}$} & \multicolumn{3}{|c|}{$\begin{array}{c}\text { Valor de la Producción destinada al autoconsumo } \\
\text { para la alimentación del hogar * }\end{array}$} \\
\hline & Importancia \% & $\begin{array}{c}\text { Por hogar } \\
\left(4,7 \text { uae) } / \mathrm{mes}^{\star *}\right.\end{array}$ & Por hogar /año** \\
\hline Cultivo en Chagras y solares & $38 \%$ & $\$ 138.135$ & $\$ 1.657 .615$ \\
\hline Pesca & $30 \%$ & $\$ 109.726$ & $\$ 1.316 .715$ \\
\hline Recolección de leña y madera & $17 \%$ & $\$ 61.762$ & $\$ 741.148$ \\
\hline Cacería & $7 \%$ & $\$ 26.229$ & $\$ 314.751$ \\
\hline $\begin{array}{l}\text { Recolección frutas } \\
\text { y semillas para artesanías }\end{array}$ & $2 \%$ & $\$ 8.744$ & $\$ 104.933$ \\
\hline Cría de animales de patio & $5 \%$ & $\$ 19.655$ & $\$ 235.855$ \\
\hline Empleos & - & - & - \\
\hline Total autoconsumo & $100 \%$ & $\$ 364.251$ & $\$ 4.371 .017$ \\
\hline \% SMLV 2007 (\$433.700 pesos/mes) & & $84 \%$ & \\
\hline
\end{tabular}

* Cálculos y proyecciones de la autora a partir de la información muestral

** No incluye el valor de los ingresos recibidos por transferencias externas o programas de seguridad social

La Tabla 4, describe la importancia económica y social que posee el recurso pesquero y la pesca artesanal como fuente de alimentación directa de los hogares del municipio, que en términos de seguridad alimentaria, contribuye con el $30 \%$ del valor económico del consumo total de los hogares, el $81 \%$ del valor de su ingesta de proteína y el $67 \%$ del volumen de ingesta de la misma.

Tabla 4. Valor del recurso pesquero destinado al autoconsumo

\begin{tabular}{|c|c|c|c|}
\hline \multirow{3}{*}{$\begin{array}{l}\text { Actividades productivas } \\
\text { generadoras de ingresos } \\
\text { en hogares ribereños }\end{array}$} & \multicolumn{3}{|c|}{ Valor de la Producción destinada al autoconsumo } \\
\hline & \multicolumn{3}{|c|}{ Fuente de proteína animal del hogar } \\
\hline & $\begin{array}{l}\text { Valor hogar / mes } \\
\text { a precios } 2007\end{array}$ & $\begin{array}{c}\% \text { valor del consumo } \\
\text { proteína }\end{array}$ & $\begin{array}{l}\text { Volumen (kg) } \\
\text { (aguas altas) }\end{array}$ \\
\hline Pesca & $\$ 109.726$ & $81 \%$ & $16.5 \mathrm{Kg}$ hogar / mes \\
\hline Cacería & $\$ 26.229$ & $19 \%$ & $8 \mathrm{Kg}$ hogar / mes \\
\hline Total & $\$ 135.955$ & $100 \%$ & $24.5 \mathrm{Kg}$ hogar / mes \\
\hline SMLV 2007 & \multicolumn{3}{|c|}{$\$ 433.700$} \\
\hline
\end{tabular}




\section{La pesca para el mercado}

Del $24 \%$ de los ingresos totales que representa la pesca, el $14 \%$ se consume directamente al interior de hogar y el restante, es decir el $10 \%$, se vende en el mercado local (ya sea en cada comunidad o en el casco urbano de Puerto Nariño). De esta forma, el comercio de recursos pesqueros aparece como la segunda fuente de ingresos monetarios que genera el $19 \%$ de los ingresos en dinero, seguido de la venta de productos agrícolas locales (producción en chagras, rastrojos y solares). Por otro lado, la población ha venido integrándose al mercado paulatinamente, tanto en la esfera comercial como laboral (Murillo, 2006; Ochoa, Wood, \& Zárate, 2006; Rodríguez \& Maldonado, 2012). La Tabla 5 deja entrever que la mayor proporción del ingreso recae sobre los recursos naturales que son responsables de garantizar el $82 \%$ de los medios para la subsistencia de la población (Valor del autoconsumo/ valor del ingreso total). La Tabla 5 presenta la composición de los ingresos monetarios de los hogares ribereños y la participación de cada actividad productiva en la economía de Puerto Nariño. Así, para los hogares de la muestra, el valor de los recursos pesqueros capturados por el grupo familiar y vendidos directamente en algún mercado cercano se estimó en $\$ 77.230$ pesos colombianos/mes por hogar, que representan el $18 \%$ de un SMLV para el año de estudio. Estos valores aunque son estables a lo largo del año, también pueden cambiar, pues como se explicó antes, la capacidad de captura y la disponibilidad del recurso pesquero, dependen directamente de la fase del ciclo hidrológico y de los ambientes pesqueros del municipio (Tabla 5).

Tabla 5. Fuentes y valores del ingreso monetario del hogar ribereño a precios 2007.

\begin{tabular}{|c|c|c|c|}
\hline \multirow{2}{*}{$\begin{array}{l}\text { Actividades productivas } \\
\text { generadoras de ingresos }\end{array}$} & \multicolumn{3}{|c|}{$\begin{array}{c}\text { Valor de la Producción para la venta como fuente } \\
\text { de ingresos monetarios del hogar* }\end{array}$} \\
\hline & Importancia \% & $\underset{\text { mes }^{\star \star}}{\text { Por hogar }}$ & Por hogar /año** \\
\hline Cultivo en chagras y solares & $25 \%$ & $\$ 106.375$ & $\$ 1.276 .501$ \\
\hline Pesca & $19 \%$ & $\$ 77.230$ & $\$ 926.758$ \\
\hline Recolección de leña y madera & $13 \%$ & $\$ 55.893$ & $\$ 670.713$ \\
\hline Cacería & $1 \%$ & $\$ 7.243$ & $\$ 86.912$ \\
\hline $\begin{array}{l}\text { Recolección frutas y semillas } \\
\text { para artesanías }\end{array}$ & 5,6 & $\$ 21.116$ & $\$ 253.389$ \\
\hline Cría de animales de patio & $2,4 \%$ & $\$ 9.175$ & $\$ 110.095$ \\
\hline Empleos & $34 \%$ & $\$ 142.405$ & $\$ 363.588$ \\
\hline Total ingreso monetario & $100 \%$ & $\$ 419.436$ & $\$ 3.687 .956$ \\
\hline $\begin{array}{l}\text { Total ingresos ambientales } \\
\text { monetarios }\end{array}$ & $66 \%$ & $\$ 277.031$ & $\$ 3.324 .272$ \\
\hline SMLV 2007 (433.700 \$ pesos/mes) & $64 \%$ & & \\
\hline
\end{tabular}

* Cálculos y proyecciones de la autora a partir de la información muestral

** No incluye el valor de los ingresos recibidos por transferencias externas o programas de seguridad social

Se observa que los ingresos monetarios totales derivados de la comercialización del medio natural (Ingresos ambientales monetarios) alcanzan los \$277.031 pesos colombianos mensuales por hogar, los cuales equivalen al 64\% de un SMLV para el año de estudio. Estas cifras permiten comprender la importancia de la gestión y conservación medioambiental como fuente de sustento y bienestar de las poblaciones rurales, campesinas, ribereñas e indígenas, que habitan estos territorios y dependen de los ecosistemas naturales para su sobrevivencia. 


\section{Contribución de la pesca artesanal a la seguridad alimentaria}

Como se ha venido presentando, el pescado y otros productos del río constituyen la principal fuente de proteína animal de la dieta alimentaria de la población ribereña (Agudelo, Sánchez, Rodríguez, Bonilla, \& Gómez, 2011; Alarcón \& Pulido, 2004; Camacho, 2012; Duque et al., 2008; Rodríguez \& Maldonado, 2009; Trujillo, 2008; Vieco \& Oyuela, 1999). Para el caso de la población amazónica de Puerto Nariño, el $100 \%$ de los hogares encuestados reportaron el consumo de pescado dentro de su dieta cotidiana, el $96 \%$ lo reporta por autoconsumo y el $4 \%$ restante (un hogar) lo compra. Si se considera el consumo total de pescado (sumando pesca directa, compra y regalo), se encuentra que en promedio, para la época de muestreo, cada hogar consumió alrededor de 1.5 sartas diarias de pescado, que equivalen a $0,67 \mathrm{~kg}$ diarios por hogar en épocas de aguas altas y $2.4 \mathrm{~kg}$ diarios por hogar en épocas de aguas bajas (Trujillo \& Trujillo, 2010); con un peso por sarta de $1.3 \mathrm{~kg}$ en aguas bajas y 0,45 $\mathrm{kg}$ en aguas altas, según lo reportado en (Ochoa, Wood \& Zárate, 2006) (Tabla 6).

Tabla 6. Ingesta promedio de pescado en los hogares indígenas de Puerto Nariño

\begin{tabular}{l|l|l}
\hline $\begin{array}{l}\text { Ingesta } \\
\text { de pescado }\end{array}$ & \multicolumn{1}{|c}{$\begin{array}{c}\text { Aguas } \\
\text { Altas }\end{array}$} & \multicolumn{1}{c}{$\begin{array}{c}\text { Aguas } \\
\text { Bajas }\end{array}$} \\
\hline $\begin{array}{l}\text { Consumo } \\
\text { del hogar }\end{array}$ & 1,5 sartas/día & 1,5 sartas /día \\
\hline $\begin{array}{l}\text { Peso Sarta } \\
\begin{array}{l}\text { Consumo } \\
\text { Hogar/ } \\
\text { Día }\end{array}\end{array}$ & $0,45 \mathrm{~kg}$ & $1,33 \mathrm{~kg}$ \\
$\begin{array}{l}\text { Consumo } \\
\text { Persona/Día }\end{array}$ & $0,67 \mathrm{~kg}$ & $2,4 \mathrm{~kg}$ \\
\hline
\end{tabular}

Es importante mencionar que este volumen de consumo se distribuye entre todos los habitantes del hogar (8 personas en promedio y 4,7 UAE), lo que implica un consumo aproximado de $84 \mathrm{~g}$ diarios por persona (para la época de estudio) que representan $2,6 \mathrm{~kg}$ al mes, $31 \mathrm{~kg}$ al año por persona y $243 \mathrm{~kg} \mathrm{al}$ año por hogar promedio, aunque como ya se mencionó, esto varía según el período del ciclo hídrico. La región de Puerto Nariño, por ser una zona que se inunda durante una parte considerable del año, no presenta una vocación ganadera y tampoco cuenta con una marcada abundancia de especies que permitan la cacería para el consumo humano; razón por la cual el consumo de carne bovina o de carne de monte, no es tan significativo en la ingesta de alimentos, en la contribución al consumo de proteína o a la economía del hogar (Trujillo, 2008). Estas cifras ratifican el papel que juegan estos sistemas en la seguridad alimentaria y la importancia de la conservación y el buen manejo del recurso en la ingesta de proteína de las poblaciones ribereñas. Según la Organización Mundial de la Salud, una persona de $70 \mathrm{~kg}$ de peso corporal requiere consumir $35 \mathrm{~g}$ de proteínas por día para suplir los requerimientos corporales y la demanda de nutrientes (FAO, 2014).

Para el caso de Puerto Nariño, se han reportado diferentes niveles de consumo e ingesta de pescado, dependiendo especialmente de la época del año, siendo este estudio el que muestra el menor volumen consumido. La diferencia en estos valores se puede originar en el período en que se realizó el muestreo (aguas en descenso), en el cual se presenta la menor disponibilidad del recurso, comparada con la época seca o de aguas en ascenso o de inundación (Duque et al., 2008), o también a la paulatina reducción del recurso ocasionada por la sobrepesca y el deterioro general de algunos ambientes acuáticos amazónicos (Camacho, 2012). Estos resultados contrastan con Fabré \& Alonso (1998) que reportan para la región del medio Amazonas, un nivel de consumo de pescado de $24 \mathrm{~kg}$ al mes por habitante, (Agudelo, Alonso, \& Sánchez, 2009), para Puerto Nariño reportan un consumo de $170 \mathrm{~g}$ diarios por persona, que representan $5,1 \mathrm{~kg}$ al mes y también para Puerto Nariño: Ochoa, Wood, \& Zárate (2006) reportan un consumo de 1,5 sartas por hogar, resultados muy similares a los obtenidos en esta investigación.

Estos resultados reflejan la importancia económica del recurso y su contribución a la calidad de vida, $y$, a su vez evidencian la dependencia de la población ribereña de la salud del ecosistema acuático y las acciones de gestión ambiental o conservación que sobre éste se desarrollen. Adicionalmente, la 
contribución económica de la pesca artesanal aquí identificada, visibiliza la disponibilidad de recursos que actualmente enfrentan las diferentes comunidades amazónicas ribereñas, el cambio en las actividades de subsistencia y la vulnerabilidad de esta sociedad ante cambios en la calidad ambiental, climática, u otros cambios globales que alteren drásticamente las dinámicas del ecosistema.

\section{Conclusiones y lecciones aprendidas}

La pesca y el uso de los recursos naturales comunes aparecen como la principal fuente de ingreso de la población, que a pesar de encontrarse en un proceso de articulación al mercado dependen ampliamente de ellos como principal estrategia de sustento. No obstante esta condición de economía de subsistencia, y la marcada dependencia de los recursos naturales, los resultados de esta investigación muestran que los ingresos reales domésticos y por tanto el bienestar de la población, se reportan por encima de los ingresos monetarios promedio de la población colombiana en las ciudades y cabeceras departamentales. Los ingresos totales del hogar (el autoconsumo del hogar y sus ingresos monetarios) generados por el medio natural, se acercan al salario mínimo mensual, lo que evidencia la importancia de salud ambiental como fuente de bienestar y como garante de la calidad de vida de estas poblaciones.

La pesca ha sido durante siglos una fuente de subsistencia y de seguridad alimentaria para las poblaciones ribereñas. Al mismo tiempo estas poblaciones han logrado organizarse y evolucionar entorno a esta actividad, constituyendo su principal fuente de ingresos y cohesión cultural. Del mismo modo, ha incidido en la estructura de la cosmovisión y la representación simbólica y cultural; concibiendo la relación entre el recurso hídrico y el recurso pesquero en un medio que los conecta con su identidad, con sus orígenes, y con el territorio. Esta dinámica ha generado una cohesión social y un arraigo cultural, el cual es transmitido y se releva de generación tras generación como fuente de conservación cultural. En consecuencia, el deterioro o la extinción de éste recurso pone en riesgo la conservación de la identidad cultural; no obstante dichas comunidades recurren a la búsqueda de otras alternativas de subsistencia.

Se resalta la importancia de la calidad ambiental y el nivel de conservación de los recursos naturales que representan la principal fuente de sustento de la población ribereña. Para garantizar un nivel de ingresos estable y adecuado a las comunidades ribereñas, se torna necesario emprender acciones encaminadas a mantener la calidad de los recursos que proveen el sustento y los medios para la reproducción social y cultural de esta población. En este sentido, la política ambiental y la gestión del recurso pesquero cobran vital importancia como fuente y medio de calidad vida para las poblaciones ribereñas.

La gestión ambiental del recurso se tornó esencial para la conservación cultural y económica de las poblaciones ribereñas; sin embargo, en el contexto amazónico colombiano prevalece la ausencia del control estatal, dadas las características departamentales donde predominan las formas de propiedad colectiva y las zonas de conservación. Bajo estas condiciones, es primordial consolidar modelos de gestión y control de recursos naturales a nivel colectivo y/o comunitario, diferentes a los sistemas estatales de controles y restricciones al uso de los recursos naturales. Estos sistemas alternativos, tales como las vedas voluntarias, la rotación de ambientes pesqueros, los acuerdos de pesca y las reservas extractivas planificadas que ya se aplican en la Amazonia colombiana, han permitido construir formas diferentes de control y aprovechamiento sostenible de los recurso colectivos en pro de mantener la oferta natural y la economía de las sociedades ribereñas.

Estos nuevos sistemas de gestión ambiental comunitaria, y la consolidación de una sociedad local organizada y capacitada para conducir la gestión de sus propios territorios, puede ser la ruta para perpetuar los diversos recursos que estos poseen y por la misma vía garantizar que estas sociedades puedan continuar con su modo de vida y su bienestar. 


\section{Agradecimientos}

Esta investigación fue desarrollada dentro del marco del proyecto de investigación, "Selva y mercado: exploración cuantitativa de los ingresos en hogares indígenas". Las autoras agradecen a las diversas personas e instituciones que apoyaron su realización. Se reconoce la participación y dedicación de los 100 hogares indígenas que recopilaron la información económica de sus hogares; a los profesores de la Maestría en Estudios Amazónicos de la Universidad Nacional de Colombia por la dirección del proyecto, y a los docentes de la Universidad del Quindío por sus valiosos comentarios en la elaboración de este artículo. También se extiende un especial agradecimiento a las instituciones que financiaron las diversas etapas del proyecto: El "Programa de Economía ambiental para América Latina y el Caribe" LACEEP-CATIE (Costa Rica), el programa "Saber y gestión ambiental en la Amazonia colombiana" NUFFIC-UNAL (Holanda) y la Fundación Omacha (Colombia-Amazonas).

\section{Literatuta Citada}

1. Agudelo, E., Alonso, J. C. \& Sánchez, L. (2009). La utilización de los recursos ícticos en la Amazonía sur de Colombia: Una estrategia de vida, ocupación y renta. In E. Agudelo, H. B. Z., \& M. Onaindia. UNESCO.

2. Agudelo, E., Sánchez, C. L., Rodríguez, C. A., Bonilla, C. A. \& Gómez, G. A. (2011). Diagnóstico de la pesquería en la cuenca del Amazonas. In I. H. Colombia, Pesquerías continentales de Colombia Vol. 2, pp. 143166. Bogotá, Colombia: Unión Gráfica.

3. Alarcón, J. \& Pulido, S. (2004). Determinación de la canasca usual de alimentos de Puerto Nariño. Tesis de grado. Bogotá: Universidad Nacional de Colombia.

4. Alcaldía de Puerto Nariño. (2008). Plan de Desarrollo 2008-2011 "Nechu: Un gobierno con identidad y autonomía". Puerto Nariño.

5. Alcaldía de Puerto Nariño. (2012). Plan de Desarrollo Municipal 2012-2015. Puerto Nariño.

6. Ardila, L. S. (2009). Dinámica de gramalotales en la llanura inundable del río Amazonas. Tesis de Maestría en Estudio Amazónicos. Leticia: Universidad Nacional de Colombia sede Amazonas.

7. Camacho, K. A. (2012). Medio Ambiente pesca y economía local: Conocimiento y territorio en áreas de la cuenta Amazónica. Tesis doctoral Facultad de Geografía e Historia. Universidad de Salamanca. Salamanca: Universidad de Salamanca.
8. Cavendish, W. (1999). Environmental resource use and the household accounts: Collection, cleaning, accounting, aggregation and headline results, a background guide for the 1993/94 dataset for Shindi Ward, Zimbabwe. London: Imperial College.

9. DANE, Departamento Administrativo Nacional de Estadística. (2011). Estudios de proyecciones de población a nivel nacional y departamental. Bogotá: DANE.

10. Deaton, A. \& Grosh, M. (1989). The influence of household compotition on household expenditure patterns: Theory and spanish evidence. Journal of Political Economy, 97(1), 179-200.

11. Domínguez, C. (2005). Amazonía Colombiana: Economía y Poblamiento. Bogotá: Universidad Externado de Colombia.

12. Duque, S. R., Trujillo, C., Ángela, H., Silvia, L., Daza, E. \& Trujillo, F. (2008). Plan de Control y Manejo de las Zonas Bajas y Sagradas para la Conservación del Pueblo Ticoya, Daiûne, Kikuri, Codi. Universidad Nacional de colombia Sede Amazonía; Fundación Omacha;Corpoamazonía, Amazonas, Leticia.

13. Durrance, M. L. (2003a). La naturaleza acuática en la vida social y cultural de los indígenas Tycunas del municipio de Puerto Nariño Amazonas. Proyecto de grado Facultad de Ciencias Sociales Universidad de los Andes. Bogotá: Departamento de Antropología Universidad de los Andes.

14. Durrance, M. L. (2003b). Pesca de consumo, cambios sociales y transformaciones en la composición de las capturas, artes y zonas de pescas en el municipio de Puerto Nariño Amazonas. Proyecto de grado Facultad de Ciencias. Bogotá: Departamento de Biología Universidad de los Andes.

15. Fabré, N. \& Alonso, J. C. (1998). Recursos ícticos Alto Amazonas: Sua importancia para as populacoes riverinas. Boletím do Museu Paraense Emilio Goeldi. , 14(1), 19-55.

16. FAO (2014). Estado mundial de la pesca y la agricultura. Roma: FAO.

17. Fernández, J. P. (1996). "El paradigma de la tragedia de los comunes y el caso de los pescadores". In M. N. Chamoux, \& J. Contreras, La Gestión comunal de recursos: economía y poder en las sociedades locales de España y América Latina. (pp. 143-164). Barcelona: Icaria.

18. Gashe, J. \& Vela, N. (2011). Sociedad bosquecina: Ensayo de antropología rural amazónica acompañado de una crítica y propuesta alternativa de propuestas de desarrollo. Instituto de Investigaciones de la Amazonía Peruana. Iquitos - Perú: Asociación Gráfica Educativa.

19. Gobernación del Amazonas. (2012). Plan de Desarrollo Departamental del Amazonas (2012-2015): Por un buen vivir somos pueblo somos más. Amazonas, Leticia.

20. Huérfano, Á. (2010). San José km 6: Transformaciones de una comunidad Tycuna del Amazonas Colombiano. Tesis de Maestría Estudios Amazónicos. Leticia: Universidad Nacional de Colombia sede Amazonía. 
21. López, S. (2008). El Pirarucú Arapaima Gigas en los lagos del sistema de Tarapoto: Aspectos demográficos y culturales. Tesis de Maestría en Estudios Amazónicos. Leticia: Universidad Nacional de Colombia Sede Amazonas.

22. Meisel, A., Bonilla, L. \& Sánchez, A. (2013, octubre ). Geografía económica de la Amazonía Colombiana. Documentos de Trabajo Sobre Economía Regional (193), 1-119.

23. Morán, R. E., Téllez, J. \& Cifuentes, J. L. (2010). "La investigación pesquera: una reflexión epistemológica" Theomai $N^{\circ} 21,97-112$.

24. Murillo, J. (2006). Percepciones de los usarios del bosque: Madereros y artesanos en torno al aprovechamiento forestal en elmunicipio de Puerto Nariño Amazonas. Tesis de grado. Bogotá: Universidad Javeriana.

25. Ochoa, G., Wood, A. \& Zárate, C. (2006). Puerto Nariño: El pueblo que se mira en el río. Retos al desarrollo sustentable en los municipios amazónicos. Bogotá, Colombia: Publicaciones ILSA.

26. Pantevis, Y. A. (2013). Construyendo la historia ambiental de Leticia a través de la pesca. Tesis de Maestría en Estudios Amazónicos. Leticia: Universidad Nacional de Colombia.

27. Pinedo, D. \& Soria, C. (2008). El Manejo de las pesquerías en ríos tropicales de Sudamérica. (I. d. Común, Ed.) Lima, Perú.

28. Rodríguez, K. J. \& Maldonado, J. H. (2012). De la extracción de madera hacia los productos forestales no maderables del bosque: Análisis de la disponibilidad de cambio en los hogares de Puerto Nariño, Amazonas, Colombia. Investigación Ambiental, 4(1), 33-44.
29. Rodríguez, K. \& Maldonado, J. H. (2009). Importancia de los productos forestales maderables y no maderables en los hogares de Puerto Nariño (AMazonas Colombia). Cuadernos de desarrollo rural, 6(62), 31-52.

30. Trujillo, C. (2008). Selva y mercado: Exploración cuantitiva de los ingreso en hogares indígenas. Tesis de Maestría en Estudio Amazónicos. Leticia, Colombia: Universidad Nacional de Colombia.

31. Trujillo, C. (2014). Pesca economía y seguridad alimentaria para el pueblo indígena de Puerto Nariño. In F. Trujillo, \& S. R. Duque, Los humedales de Tarapoto aportes al conocimiento sobre su biodiversidad y uso (pp. 260-293). Bogotá, Colombia: Unión Gráfica.

32. Trujillo, C. \& Escobar, A. L. (2015). Restricciones conceptuales del ordenamiento territorial Colombiano: Usos del territorio y formas de propiedad. Entramado, 11(2), 20-36.

33. Trujillo, C. \& Trujillo, F. (2010). Acuerdos de pesca responsable para el buen uso de los lagos de Tarapoto. Bogotá: Unión Gráfica.

34. Trujillo, F. \& Duque, S. R. (2014). Los humadales de Tarapoto, aportes al conocimiento sobre su biodiversidad y uso . Bogotá: Unión Gráfica.

35. Vieco, J. J. \& Oyuela, A. (1999). La pesca entre los tycunas: Historia, técnicas y ecosistemas. Boletín de Antropología volúmen $13 N^{\circ}$. 30 . .

36. Zamora, A., Narváez, J. \& Londoño, L. M. (2007). Evaluación Económica de la Pesquería Artesanal de la Ciénaga Grande de Santa Martha y Complejo de Pajarales-Caribe Colombiano. (I. d. INVEMAR, Ed.) Boletín de Investigaciones Marinas y Costeras, 33-48.
Conflicto de Intereses

Los autores declaran no tener ningún conflicto de intereses

Recibido: 16 de mayo de 2015 Aceptado: 06 de julio de 201 
